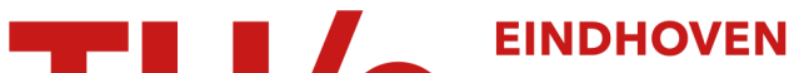 UNIVERSITY OF TECHNOLOGY
}

\section{On the synthesis of boundary control laws for distributed port- hamiltonian systems}

\section{Citation for published version (APA):}

Macchelli, A., Le Gorrec, Y., Ramirez, H., \& Zwart, H. (2017). On the synthesis of boundary control laws for distributed port-hamiltonian systems. IEEE Transactions on Automatic Control, 62(4), 1700-1713. [7524022]. https://doi.org/10.1109/TAC.2016.2595263

\section{Document license:}

TAVERNE

DOI:

10.1109/TAC.2016.2595263

Document status and date:

Published: 01/04/2017

\section{Document Version:}

Publisher's PDF, also known as Version of Record (includes final page, issue and volume numbers)

\section{Please check the document version of this publication:}

-A submitted manuscript is the version of the article upon submission and before peer-review. There can be important differences between the submitted version and the official published version of record. People interested in the research are advised to contact the author for the final version of the publication, or visit the $\mathrm{DOI}$ to the publisher's website.

- The final author version and the galley proof are versions of the publication after peer review.

- The final published version features the final layout of the paper including the volume, issue and page numbers.

Link to publication

\section{General rights}

Copyright and moral rights for the publications made accessible in the public portal are retained by the authors and/or other copyright owners and it is a condition of accessing publications that users recognise and abide by the legal requirements associated with these rights.

- Users may download and print one copy of any publication from the public portal for the purpose of private study or research.

- You may not further distribute the material or use it for any profit-making activity or commercial gain

- You may freely distribute the URL identifying the publication in the public portal.

If the publication is distributed under the terms of Article $25 \mathrm{fa}$ of the Dutch Copyright Act, indicated by the "Taverne" license above, please follow below link for the End User Agreement:

www.tue.nl/taverne

\section{Take down policy}

If you believe that this document breaches copyright please contact us at:

openaccess@tue.nl

providing details and we will investigate your claim. 


\title{
On the Synthesis of Boundary Control Laws for Distributed Port-Hamiltonian Systems
}

\author{
Alessandro Macchelli, Member, IEEE, Yann Le Gorrec, Member, IEEE, \\ Héctor Ramírez, Member, IEEE, and Hans Zwart, Member, IEEE
}

\begin{abstract}
This paper is concerned with the energy shaping of 1-D linear boundary controlled port-Hamiltonian systems. The energy-Casimir method is first proposed to deal with power preserving systems. It is shown how to use finite dimensional dynamic boundary controllers and closed-loop structural invariants to partially shape the closed-loop energy function and how such controller finally reduces to a state feedback. When dissipative port-Hamiltonian systems are considered, the Casimir functions do not exist anymore (dissipation obstacle) and the immersion (via a dynamic controller)/reduction (through invariants) method cannot be applied. The main contribution of this paper is to show how to use the same ideas and state functions to shape the closed-loop energy function of dissipative systems through direct state feedback i.e. without relying on a dynamic controller and a reduction step. In both cases, the existence of solution and the asymptotic stability (by additional damping injection) of the closed-loop system are proven. The general theory and achievable closed-loop performances are illustrated with the help of a concluding example, the boundary stabilization of a longitudinal beam vibrations.
\end{abstract}

Index Terms-Boundary control, distributed port-Hamiltonian systems, passivity-based control, stability of pdes.

\section{INTRODUCTION}

$\mathbf{I}$ $\mathrm{T}$ is more than two centuries that partial differential equations (PDEs) are used to model physical systems. However, one of the most recurring assumption is that no external signals are present. In this respect, it is only since the sixties and seventies of the last century that a mathematical theory has been developed in order to cope with boundary control and observation. This fact makes it possible to study practical problems modeled by PDEs, such as controlling the water level in a river, or estimating the temperature distribution in a room.

Manuscript received April 8, 2016; revised June 27, 2016; accepted July 8, 2016. Date of publication July 27, 2016; date of current version March 27, 2017. This work was supported by French ANR sponsored projects HAMECMOPSYS and Labex ACTION under reference codes ANR-11-BS03-0002 and ANR-11-LABX-01-01, respectively. Recommended by Associate Editor M. Opmeer.

A. Macchelli is with the Department of Electrical, Electronic and Information Engineering "Guglielmo Marconi" (DEI), University of Bologna, 40136 Bologna, Italy (e-mail: alessandro.macchelli@unibo.it).

$\mathrm{Y}$. Le Gorrec and $\mathrm{H}$. Ramírez are with the FEMTO-ST Institute, AS2M Department, University of Bourgogne-Franche-Comté/CNRS, F-25000 Besançon, France (e-mail: legorrec@femto-st.fr; hector. ramirez@femto-st.fr).

H. Zwart is with the Department of Applied Mathematics, University of Twente, 7500 AE Enschede, The Netherlands, and also with the Department of Mechanical Engineering, Eindhoven University of Technology, 5600 MB Eindhoven, The Netherlands (e-mail: h.j.zwart@utwente.nl).

Color versions of one or more of the figures in this paper are available online at http://ieeexplore.ieee.org.

Digital Object Identifier 10.1109/TAC.2016.2595263
Moreover, by introducing inputs and outputs, the distributed parameter system is no longer a "closed" system since it can be easily interconnected with other (sub-)systems.

From a physical point of view and with the bond-graph modeling formalism [1] in mind, the interaction between different systems can be interpreted as an exchange of energy through a set of well-defined power ports. Port-Hamiltonian systems [2], [3] have been introduced about 20 years ago as the mathematical formalization of bond-graphs to describe lumped parameter physical systems in an unified manner, [4], [5]. The generalization to the infinite dimensional scenario leads to the definition of distributed port-Hamiltonian systems [6]-[9] that have been introduced about one decade ago as a particular case of the more general framework presented, e.g., in [10], that deals with closed infinite dimensional Hamiltonian systems, and then extended in [11] (see also the references therein), to open physical systems. Distributed port-Hamiltonian systems have proved to represent a powerful framework for modeling, simulation and control of physical systems described by PDEs.

Most of the current research on the stabilization of distributed port-Hamiltonian systems is about the development of boundary controllers. The simplest way of designing such controllers is to add some dissipation, or to use the passivity properties of the interconnected systems and the total energy as Lyapunov function to prove asymptotic or exponential stability. Inspired by the finite dimensional case, a more sophisticated approach aiming at achieving a certain level of performances in closedloop consists in shaping the energy function, the stability being ensured by the passivity properties of the controlled system. In current literature (see e.g., [11]-[16]), this task has been accomplished for power preserving systems by considering a dynamic controller and generating a set of closed-loop Casimir functions that relates the state of the infinite dimensional plant to the state of the finite dimensional controller. The shape of the closed-loop energy function is then changed by acting on the Hamiltonian of the controller. From the existence of the closed-loop structural invariants, the dynamic controller finally reduces at the end to a state feedback. This procedure is the generalization of the control by interconnection (energyCasimir method or immersion/reduction methods) developed for finite dimensional systems, [3], [17]. The strong limitation of such control design method is the dissipation that breaks the structural invariants. This phenomenon is well known as the dissipation obstacle.

This paper focuses on the class of distributed portHamiltonian systems defined on real Hilbert spaces studied in [8], [18], where the problem of existence of solutions for the associated system of PDEs, and of the selection of the 
boundary conditions to have a well-defined boundary control system in the sense of [19] has been solved in case of linear systems with one-dimensional spatial domain. The latest results that combine abstract functional analytical approach with the physical approach of port-Hamiltonian system theory have been collected in [20], in which, among others, simple matrix conditions for well-posedness and stability are given. With the framework proposed in [8], [20] in mind, in this paper new results dealing with the synthesis of asymptotically stabilizing boundary control laws are given.

The starting point is the energy-Casimir method, here investigated in the most general possible case as far as the controller structure is concerned. In this way, the results already presented in literature [11]-[16] can be seen as particular cases of the theory discussed here. In first instance, the geometric properties of the closed-loop system are investigated. General conditions for the existence of Casimir functions are provided, together with a precise characterisation of the class of systems to which the method is applicable. It is well-known, in fact, that with this approach it is not possible to deal with systems that are characterised by equilibria which require an infinite amount of supplied energy in steady state, i.e., with the so-called "dissipation obstacle," [3], [15], [17], [21]-[23]. Secondly, based on [24]-[27], existence and properties of the closed-loop system solutions are investigated, and a positive answer in case the controller is passive is given. Once the Casimir functions are characterized, it is shown how to use them for control purposes. Indeed, these invariants allow to link the state of the controller to the state of the system, and then to reduce the dynamic contribution of the controller to a boundary state feedback. An appropriate choice of this state feedback through the initial choice of the controller energy function allows to shape, at least in some directions (this point is discussed in the last section of the paper), the closed-loop energy function. Such a control action can be paired, for example, with damping injection without worrying that possible changes in the dissipative structure of the system "destroy" the Casimir functions, thus ensuring, after having proved existence of solution, the asymptotic stability of the closed-loop system.

Inspired by this energy-Casimir method, a new control design method is proposed to avoid the problems associated to the dissipation obstacle. The idea is to keep a boundary state feedback structure without designing it through a dynamic controller nor closed-loop invariants. In this paper, all the boundary state feedback laws that shape the Hamiltonian function in predefined directions are characterised, so that simple stability in closed-loop is obtained. To have asymptotic stability, it is then necessary to add damping by means of a further control loop. This is the same concept adopted in finite dimensions in case of stabilization with state modulated sources [17], or with the more general IDA-PBC control technique, [28]. These considerations lead to the last main contribution of this paper. It is shown that if it is possible, via damping injection, to impose full boundary dissipation to the closed-loop port-Hamiltonian systems with shaped Hamiltonian, then the desired equilibrium is asymptotically stable.

It is now important to understand how to frame this work in the more general topic "control of distributed parameter systems." First of all, there are several sub-classes of infinitedimensional systems. In the general operator-theoretic approach [19] the use of energy is most times hidden, although the co-located feedback is based on it. Therefore, our approach is more related to the second main subclass of infinitedimensional systems, namely working with the PDE directly, [29]. In this class, the use of energy is very common. However, in this paper, there is no reference to a specific PDE, but to a class of PDEs that encompasses models, e.g., of flexible beams, waves, and reaction diffusion processes, in 1-D but also 2-D or 3-D when there are symmetries that can be exploited to obtain a simplified 1-D model, and that forms a sub-class of port-Hamiltonian systems. Furthermore, the idea is not to stabilize the system around the origin (the lowest point of the energy), but around another point with modified closed-loop performances associated to a modified shape of the closed-loop energy. Combined with the analytic proof that this is possible, to the best of our knowledge this has not been studied before neither in the operator approach, nor in the PDE approach.

The paper is organized as follows. In Section II, the class of linear, distributed, port-Hamiltonian systems under investigation is briefly presented. In Section III, the geometric properties of the energy-Casimir method are discussed. Section IV is devoted to the main control synthesis methodology that is based on passivity-based considerations. How to achieve asymptotic stability via damping injection is then discussed in Section V. Finally, in Section VI, the general methodology is illustrated with the help of an example, namely the PDE that describes the longitudinal vibration of a beam. Conclusions and a discussion about possible future research activities are reported in Section VII.

\section{BACKGROUND}

In this paper, we refer to the class of linear distributed portHamiltonian systems defined on real Hilbert spaces that have been studied in [8], [20], [27], [30], i.e., to systems described by the PDE

$$
\frac{\partial x}{\partial t}(t, z)=P_{1} \frac{\partial}{\partial z}(\mathcal{L}(z) x(t, z))+\left(P_{0}-G_{0}\right) \mathcal{L}(z) x(t, z)
$$

with $x \in \mathbb{R}^{n}$, and $z \in[a, b]$. Moreover, $P_{1}=P_{1}^{T}$ and invertible, $P_{0}=-P_{0}^{T}, G_{0}=G_{0}^{T} \geq 0$, and $\mathcal{L}(\cdot)$ is a bounded and Lipschitz continuous matrix-valued function such that $\mathcal{L}(z)=$ $\mathcal{L}^{T}(z)$ and $\mathcal{L}(z) \geq \kappa I$, with $\kappa>0$, for all $z \in[a, b]$. For the sake of clearness, $(\mathcal{L} x)(t, z):=\mathcal{L}(z) x(t, z)$. We say that the symmetric matrix $M$ is positive definite, in short $M>0$, if all its eigenvalues are positive, and positive semi-definite, in short $M \geq 0$, if its eigenvalues are non-negative. The state space is $X=L^{2}\left(a, b ; \mathbb{R}^{n}\right)$, and is endowed with the inner product $\left\langle x_{1} \mid x_{2}\right\rangle_{\mathcal{L}}=\left\langle x_{1} \mid \mathcal{L} x_{2}\right\rangle$ and norm $\left\|x_{1}\right\|_{\mathcal{L}}^{2}=\left\langle x_{1} \mid x_{1}\right\rangle_{\mathcal{L}}$, where $\langle\cdot \mid \cdot\rangle$ denotes the natural $L^{2}$-inner product. The selection of this space for the state variable is motivated by the fact that $\|\cdot\|_{\mathcal{L}}^{2}$ is strongly linked to the energy function of (1). As a consequence, $X$ is also called the space of energy variables, and $\mathcal{L} x$ denote the co-energy variables. This class is quite general and includes models of flexible structures, traveling waves [7], [9], [13], heat 
exchangers, and linearised models of bio or chemical reactors among others, [31].

Remark 2.1: Note that $\mathcal{L}(\cdot)$ may be $L^{\infty}$, i.e., a bounded measurable matrix-valued function. Lipschitz continuity is only needed in the proof of Theorem 5.3.

The PDE (1) can be also written as $\dot{x}=\mathcal{J} x$, where $\mathcal{J}$ is the linear operator defined as $\mathcal{J} x:=P_{1}(\partial / \partial z)(\mathcal{L} x)+\left(P_{0}-\right.$ $\left.G_{0}\right) \mathcal{L} x$, with domain $D(\mathcal{J})=\left\{\mathcal{L} x \in H^{1}\left(a, b ; \mathbb{R}^{n}\right)\right\}$. Here, $H^{1}\left(a, b ; \mathbb{R}^{n}\right)$ denotes the Sobolev space of order one.

To have a distributed port-Hamiltonian system, the PDE (1) has to be completed by a set of boundary port variables. More precisely, for $\mathcal{L} x \in H^{1}\left(a, b ; \mathbb{R}^{n}\right)$, the boundary port variables associated to (1) are the vectors $f_{\partial}, e_{\partial} \in \mathbb{R}^{n}$ defined by

$$
\left(\begin{array}{l}
f_{\partial} \\
e_{\partial}
\end{array}\right)=\underbrace{\frac{1}{\sqrt{2}}\left(\begin{array}{cc}
P_{1} & -P_{1} \\
I & I
\end{array}\right)}_{=: R}\left(\begin{array}{l}
(\mathcal{L} x)(b) \\
(\mathcal{L} x)(a)
\end{array}\right) .
$$

The boundary port variables are a linear combination of the restriction of the co-energy variables to the boundary, and integration by parts shows that $(1 / 2)(d / t)\|x(t)\|_{\mathcal{L}}^{2}=e_{\partial}^{T}(t) f_{\partial}(t)$. The problem of determining the "right" boundary inputs and outputs for (1) to have a boundary control system on $X$ in the sense of the semigroup theory, see, e.g., [19], has been addressed in [8].

Theorem 2.1: Let $W$ be a $n \times 2 n$ real matrix. With this $W$, we define the input mapping $\mathcal{B}: H^{1}\left(a, b ; \mathbb{R}^{n}\right) \rightarrow \mathbb{R}^{n}$ and the input $u(t)$ as

$$
u(t)=W\left(\begin{array}{l}
f_{\partial}(t) \\
e_{\partial}(t)
\end{array}\right)=: \mathcal{B} x(t) .
$$

If $W$ has full rank and satisfies $W \Sigma W^{T} \geq 0$, with

$$
\Sigma=\left(\begin{array}{ll}
0 & I \\
I & 0
\end{array}\right)
$$

then the system (1) with input (3) is a boundary control system on $X$. Furthermore, the operator $\overline{\mathcal{J}} x:=P_{1}(\partial / \partial z)(\mathcal{L} x)+$ $\left(P_{0}-G_{0}\right) \mathcal{L} x$ with domain

$$
\begin{aligned}
D(\overline{\mathcal{J}}) & =\left\{\mathcal{L} x \in H^{1}\left(a, b ; \mathbb{R}^{n}\right) \mid\left(\begin{array}{l}
f_{\partial} \\
e_{\partial}
\end{array}\right) \in \operatorname{Ker} W\right\} \\
& =\left\{\mathcal{L} x \in H^{1}\left(a, b ; \mathbb{R}^{n}\right) \mid \mathcal{B} x=0\right\}
\end{aligned}
$$

generates a contraction semigroup on $X$. Moreover, let $\tilde{W}$ be a full rank $n \times 2 n$ matrix such that $\left(W^{T} \quad \tilde{W}^{T}\right)$ is invertible and let $P$ be given by

$$
P=\left(\begin{array}{ll}
W \Sigma W^{T} & W \Sigma \tilde{W}^{T} \\
\tilde{W} \Sigma W^{T} & \tilde{W} \Sigma \tilde{W}^{T}
\end{array}\right)^{-1}
$$

Define the output as

$$
y(t)=\tilde{W}\left(\begin{array}{l}
f_{\partial}(t) \\
e_{\partial}(t)
\end{array}\right)=: \mathcal{C} x(t)
$$

with $\mathcal{C}: H^{1}\left(a, b ; \mathbb{R}^{n}\right) \rightarrow \mathbb{R}^{n}$. Then, for $u \in C^{2}\left(0, \infty ; \mathbb{R}^{n}\right)$ and $(\mathcal{L} x)(0) \in H^{1}\left(a, b ; \mathbb{R}^{n}\right)$, the following energy balance equation is satisfied:

$$
\frac{1}{2} \frac{d}{d t}\|x(t)\|_{\mathcal{L}}^{2} \leq \frac{1}{2}\left(\begin{array}{l}
u(t) \\
y(t)
\end{array}\right)^{T} P\left(\begin{array}{l}
u(t) \\
y(t)
\end{array}\right) .
$$

Proof: See [8].

In this paper, the matrices $W$ and $\tilde{W}$ are selected in such a way that (1) is in impedance form i.e., $W \Sigma W^{T}=\tilde{W} \Sigma \tilde{W}^{T}=0$ and $W \Sigma \tilde{W}^{T}=I$, or equivalently

$$
\left(\begin{array}{l}
W \\
\tilde{W}
\end{array}\right) \Sigma\left(\begin{array}{ll}
W^{T} & \tilde{W}^{T}
\end{array}\right)=\Sigma .
$$

In this case, the energy-balance (5) reduces to

$$
\frac{1}{2} \frac{d}{d t}\|x(t)\|_{\mathcal{L}}^{2} \leq y^{T}(t) u(t) .
$$

In Sections IV and V, the design of a state-feedback law for the PDE (1) that leads to a closed-loop system in portHamiltonian form which is asymptotically stable is discussed. However, preliminary problems are to understand if the linear system of coupled PDEs and ODEs associated to the closedloop system has a unique solution, and if it is a well-defined boundary control system. In this respect, let us consider a linear control system in port-Hamiltonian form, whose most general formulation is [32]

$$
\left\{\begin{array}{l}
\dot{x}_{C}=\left(J_{C}-R_{C}\right) Q_{C} x_{C}+\left(G_{C}-P_{C}\right) u_{C} \\
y_{C}=\left(G_{C}+P_{C}\right)^{T} Q_{C} x_{C}+\left(M_{C}+S_{C}\right) u_{C}
\end{array}\right.
$$

where $x_{C} \in \mathbb{R}^{n_{C}}$ and $u_{C}, y_{C} \in \mathbb{R}^{n}$, while $J_{C}=-J_{C}^{T}, M_{C}=$ $-M_{C}^{T}, R_{C}=R_{C}^{T}$, and $S_{C}=S_{C}^{T}$, with this further condition satisfied

$$
\left(\begin{array}{ll}
R_{C} & P_{C} \\
P_{C}^{T} & S_{C}
\end{array}\right) \geq 0
$$

Finally, assume that $Q_{C}=Q_{C}^{T}>0$, so that (8) is a passive linear system. For the sake of compactness, this system can be easily written in standard $\left(A_{C}, B_{C}, C_{C}, D_{C}\right)$ form, being

$$
\begin{aligned}
& A_{C}=\left(J_{C}-R_{C}\right) Q_{C} \quad B_{C}=G_{C}-P_{C} \\
& C_{C}=\left(G_{C}+P_{C}\right)^{T} Q_{C} \quad D_{C}=M_{C}+S_{C} .
\end{aligned}
$$

The control system (8) is interconnected to the boundary of (1) in a power-conserving way through the input $u$ and the output $y$ defined in (3) and (4) under the assumptions (6) as

$$
\left(\begin{array}{l}
u \\
y
\end{array}\right)=\left(\begin{array}{cc}
0 & -I \\
I & 0
\end{array}\right)\left(\begin{array}{l}
u_{C} \\
y_{C}
\end{array}\right)+\left(\begin{array}{l}
u^{\prime} \\
0
\end{array}\right)
$$

where $u^{\prime} \in \mathbb{R}^{n}$ is an additional control input. This is the standard feedback interconnection. The closed-loop system is characterized by the total Hamiltonian

$$
H_{\mathrm{cl}}\left(x(t), x_{C}(t)\right)=\frac{1}{2}\|x(t)\|_{\mathcal{L}}^{2}+\underbrace{\frac{1}{2} x_{C}^{T}(t) Q_{C} x_{C}(t)}_{=: H_{C}\left(x_{C}(t)\right)}
$$


and can be compactly written as

$$
\left\{\begin{array}{l}
\dot{\zeta}=\mathcal{J}_{\mathrm{cl}} \zeta \\
u^{\prime}=\left(\mathcal{B}+D_{C} \mathcal{C} \quad C_{C}\right) \zeta=: \mathcal{B}^{\prime} \zeta
\end{array}\right.
$$

where

$$
\zeta=\left(\begin{array}{c}
x \\
x_{C}
\end{array}\right) \in Z:=X \times \mathbb{R}^{n_{C}}
$$

is the state variable of the closed-loop system and $\mathcal{J}_{\mathrm{cl}}$ : $D\left(\mathcal{J}_{\mathrm{cl}}\right) \subset Z \rightarrow Z$ is the following linear operator:

$$
\mathcal{J}_{\mathrm{cl}} \zeta:=\left(\begin{array}{cc}
\mathcal{J} & 0 \\
B_{C} \mathcal{C} & A_{C}
\end{array}\right)\left(\begin{array}{c}
x \\
x_{C}
\end{array}\right)
$$

with domain

$$
D\left(\mathcal{J}_{\mathrm{cl}}\right)=D(\mathcal{J}) \times \mathbb{R}^{n_{C}}
$$

$Z$ is endowed with the inner product defined as

$$
\left\langle\zeta_{1} \mid \zeta_{2}\right\rangle_{Z}=\left\langle x_{1} \mid x_{2}\right\rangle_{\mathcal{L}}+x_{C, 1}^{T} Q_{C} x_{C, 2}
$$

which means that $H_{\mathrm{cl}}(\zeta)=(1 / 2)\|\zeta\|_{Z}^{2}$. Some fundamental properties associated to the PDEs and ODEs describing the closed-loop dynamics are presented in the next proposition.

Proposition 2.2: Consider the port-Hamiltonian system resulting from the power-conserving interconnection (11) of (1) and (8), which results in (13). Then, (13) with $\mathcal{J}_{\mathrm{cl}}$ defined in (14) with domain (15) is a boundary control system. Moreover, the operator $\overline{\mathcal{J}}_{\text {cl }}$ given by

$$
\overline{\mathcal{J}}_{\mathrm{cl}} \zeta:=\left(\begin{array}{cc}
\mathcal{J} & 0 \\
B_{C} \mathcal{C} & A_{C}
\end{array}\right)\left(\begin{array}{c}
x \\
x_{C}
\end{array}\right)
$$

with domain

$$
D\left(\overline{\mathcal{J}}_{\mathrm{cl}}\right)=\left\{\left(\begin{array}{c}
x \\
x_{C}
\end{array}\right) \in Z \mid x \in D(\mathcal{J}), \text { and } \mathcal{B}^{\prime}\left(\begin{array}{c}
x \\
x_{C}
\end{array}\right)=0\right\}
$$

with $\mathcal{B}^{\prime}$ defined in (13) generates a contraction semigroup.

Proof: The proof can be found in [26].

\section{StRUCTURAL INVARIANTS OF BOUNDARY CONTROLLED SYSTEMS}

Proposition 2.2: Shows that the power conserving interconnection (11) of the distributed port-Hamiltonian system (1) with the passive port-Hamiltonian controller (8) results in a port-Hamiltonian system, the closed-loop system, characterized by the Hamiltonian (12) which is the sum of the Hamiltonian functions of (1) and (8). To use this closed-loop Hamiltonian as Lyapunov function, one has first to guarantee that this function has a minimum at the desired equilibrium with a proper choice of $H_{C}$. The choice of $H_{C}$ also allows to change the shape (at least in some directions) of the closed-loop energy function, and thus the closed-loop performances. As in the finite dimensional case [3], [17], if it is possible to find structural invariants (i.e., that do not depend on the Hamiltonian) named Casimir functions of the form $C\left(x, x_{C}\right)=x_{C}-F(x)$, with $F(x)$ some smooth well defined functional of $x$, then on every invariant manifold defined by $x_{C}-F(x)=\kappa$, with $\kappa$ a real constant relating the initial state of the system to the initial state of the controller, the closed-loop Hamiltonian (12) may be written as $H_{\mathrm{cl}}(x)=H(x)+H_{C}(F(x)+\kappa)$, with $H(x)=(1 / 2)\|x\|_{\mathcal{L}}^{2}$. Hence, the closed-loop Hamiltonian $H_{\mathrm{cl}}$ depends on the state variable of (1) only. Its minimum and its shape, defining the closed-loop equilibrium and the closed-loop performances, can be assigned by an appropriate choice of $H_{C}$.

Definition 3.1 (Casimir function): Consider the boundary control system defined in Proposition 2.2 with $u^{\prime}=0$ in (11). A function $C: X \times \mathbb{R}^{n_{C}} \rightarrow \mathbb{R}$ is a Casimir function if $\dot{C}=0$ along the (classical) solutions for every possible choice of $\mathcal{L}(\cdot)$ and $Q_{C}$, [3], [13], [21].

Due to the fact that the geometric structure (namely, the Dirac structure) associated to the boundary control system introduced in Proposition 2.2 is linear, the Casimir functions are linear (see e.g., [22]). Consequently, as in [26], [33], [34], we look for Casimir functions in the form

$$
C\left(x(t), x_{C}(t)\right)=\Gamma^{T} x_{C}(t)+\int_{a}^{b} \Psi^{T}(z) x(t, z) d z
$$

with $\Gamma \in \mathbb{R}^{n_{C}}$ and $\Psi \in L^{2}\left(a, b ; \mathbb{R}^{n}\right)$. Note that they are not (yet) in the form assumed above.

Proposition 3.1: Consider the boundary control system introduced in Proposition 2.2 with $u^{\prime}=0$ in (11). Then, (16) is a Casimir function for this system if and only if $\Psi \in$ $H^{1}\left(a, b ; \mathbb{R}^{n}\right)$,

$$
\begin{aligned}
& P_{1} \frac{d \Psi}{d z}(z)+\left(P_{0}+G_{0}\right) \Psi(z)=0 \\
& \left(J_{C}+R_{C}\right) \Gamma+\left(G_{C}+P_{C}\right) \tilde{W} R\left(\begin{array}{l}
\Psi(b) \\
\Psi(a)
\end{array}\right)=0 \\
& \left(G_{C}-P_{C}\right)^{T} \Gamma+\left[W+\left(M_{C}-S_{C}\right) \tilde{W}\right] R\left(\begin{array}{l}
\Psi(b) \\
\Psi(a)
\end{array}\right)=0
\end{aligned}
$$

Proof: The proof of [26], for the case $R_{C}=P_{C}=$ $M_{C}=0$, can be easily adjusted to show the above proposition. However, in Appendix A we present a simpler and more elegant one.

It is worth noting that Casimir functions are also discussed in [10] for Hamiltonian systems, and there called "distinguished functionals." They are employed in the stabilisation by portinterconnection in [11], where a finite dimensional Hamiltonian control system is interconnected to the boundary of an infinite dimensional Hamiltonian plant, and similar results to the ones in Proposition 3.1 are obtained.

Proposition 3.2: Assume that it is possible to find $n_{C}$ Casimir functions, i.e. it is possible to relate all the state variables of the controller with the states of the plant, and denote by $\hat{\Gamma}=\left(\begin{array}{llll}\Gamma_{1} & \ldots & \Gamma_{n_{C}}\end{array}\right)$ and $\hat{\Psi}=\left(\begin{array}{lll}\Psi_{1} & \ldots & \Psi_{n_{C}}\end{array}\right)$ the $n_{C} \times n_{C}$ matrices built from the vectors and vector valued functions that appear in the Casimir (16). Moreover, assume that 
the $\Psi_{i}$ are independent solutions of (17). Then, the following conditions are satisfied:

$$
\begin{aligned}
& G_{0} \hat{\Psi}(z)=0
\end{aligned}
$$

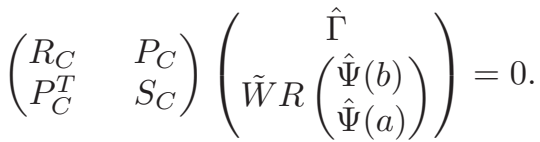

Proof: The proof is reported in Appendix B.

Propositions 3.1 and 3.2: Summarise the conditions for the existence of the Casimir invariants in closed-loop. Relations (20) and (21) impose conditions on the parameters in the Casimir when there is dissipation in the system. This is called the dissipation obstacle. For instance, when $G_{0}$ is invertible, i.e., there is strong dissipation in the PDE, (20) implies that $\hat{\Psi}$ must be zero, and so we cannot find any Casimir function of the form (16). Hence, our control design procedure fails. See also [3], [17] for the finite-dimensional case.

\section{BOUNDARY CONTROL BY ENERGY-SHAPING}

The aim of this section is to present a boundary control law able to shape the Hamiltonian and move the minimum to the desired equilibrium state. The synthesis technique discussed here allows to overcome the main limitation of the energy-Casimir method, namely the dissipation obstacle, that imposes strong constraints on the amount of damping that can be added in the system, damping that is fundamental to achieve asymptotic or exponential stability in closed-loop. Before presenting the main result of this section, it is important to investigate what is the effect of the control system (8) developed according to the energy-Casimir method on the distributed parameter system (1).

The link between the state of the controller $x_{C}$ and the state of the plant $x$ appears through the Casimir functions (16). Indeed, under the hypothesis of Proposition 3.2 and if $\hat{\Gamma}$ is invertible, since each Casimir function is constant along the system trajectories, we have that

$$
x_{C}(t)=-\hat{\Gamma}^{-T} \int_{a}^{b} \hat{\Psi}^{T}(z) x(t, z) d z+\kappa
$$

with $\kappa \in \mathbb{R}^{n_{C}}$ a constant that depends on the initial conditions only. If we assume that the controller initial state is selected in such a way that $\kappa=0$, it is possible to verify that the closedloop dynamics are given by the boundary control system

$$
\begin{aligned}
\frac{\partial x}{\partial t}(t, z) & =P_{1} \frac{\partial}{\partial z} \frac{\delta H_{\mathrm{cl}}}{\delta x}(x(t))(z)+\left(P_{0}-G_{0}\right) \frac{\delta H_{\mathrm{cl}}}{\delta x}(x(t))(z) \\
u^{\prime}(t) & =W^{\prime} R\left(\begin{array}{c}
\left(\frac{\delta H_{\mathrm{cl}}}{\delta x}(x(t))\right)(b) \\
\left(\frac{\delta H_{\mathrm{cl}}}{\delta x}(x(t))\right)(a)
\end{array}\right)
\end{aligned}
$$

in which $\delta$ denotes the functional derivative (Fréchet derivative, in the language of functional analysis) [7], [9], [10], while

$$
\begin{aligned}
H_{\mathrm{cl}}(x(t))=\frac{1}{2}\|x(t)\|_{\mathcal{L}}^{2} & +\frac{1}{2}\left(\int_{a}^{b} \hat{\Psi}^{T}(z) x(t, z) d z\right)^{T} \\
& \times \hat{\Gamma}^{-1} Q_{C} \hat{\Gamma}^{-T} \int_{a}^{b} \hat{\Psi}^{T}(z) x(t, z) d z
\end{aligned}
$$

and $W^{\prime}$ is a $n \times 2 n$ full rank, real matrix such that $W^{\prime} \Sigma W^{\prime^{T}} \geq 0$.

The fact that the closed-loop energy as function of the $x$ coordinates is given by (24) is an immediate consequence of (12) and (22) if $\kappa=0$. Moreover, the PDE that describes the closed-loop dynamics in (23) follows from the fact that

$$
\frac{\delta H_{\mathrm{cl}}}{\delta x}(z)=(\mathcal{L} x)(z)+\hat{\Psi}(z) \hat{\Gamma}^{-1} Q_{C} \hat{\Gamma}^{-T} \int_{a}^{b} \hat{\Psi}^{T}(z) x(z) d z
$$

and because from (17) and (20) we have that

$$
\begin{aligned}
0 & =P_{1} \frac{d \hat{\Psi}}{d z}(z)+\left(P_{0}+G_{0}\right) \hat{\Psi}(z)-2 G_{0} \hat{\Psi}(z) \\
& =P_{1} \frac{d \hat{\Psi}}{d z}(z)+\left(P_{0}-G_{0}\right) \hat{\Psi}(z)
\end{aligned}
$$

with the integral term that appears in the previous expression of $\delta H_{\mathrm{cl}} / \delta x$ that is not a function of $z$. Finally, with simple calculations it is possible to prove that $W^{\prime}=W+\left(M_{C}+S_{C}\right) \tilde{W}$, which from (6) and (9) implies that $W^{\prime} \Sigma W^{\prime T}=2 S_{C} \geq 0$.

The effect of the controller (8) is then to shape the openloop Hamiltonian $(1 / 2)\|x(t)\|_{\mathcal{L}}^{2}$ into the desired one (24), as expected, and this property is strictly related to the presence of Casimir functions in closed-loop that establish the algebraic relation (22) between state of the controller and of the plant. The same result can be equivalently achieved by writing the control action, i.e., the output $y_{C}$ of (8), in state-feedback form by defining $x_{C}$ as in (22), with $\kappa=0$. With such control action, the closed-loop system evolves according to (23), i.e., with the shaped Hamiltonian. Proposition 2.2 assures that also when the boundary control action is in standard state feedback form, the closed-loop system is well-posed. Furthermore, it is possible to act on the auxiliary input $u^{\prime}$ e.g., to add damping without losing the stability properties obtained in the inner loop.

Similarly to the finite dimensional case, the main contribution of this section is to use state feedback to avoid the intrinsic drawbacks of the energy-Casimir method in presence of the dissipation obstacle. In the following proposition, it is shown how to design a boundary state feedback control that is able to map the open-loop dynamics (1) into the target system given in (23).

Proposition 4.1 (Energy-shaping): Consider the system (1) with boundary control given by (3). Denote by $H(x)=$ $(1 / 2)\|x\|_{\mathcal{L}}^{2}$ its Hamiltonian function. Then, the feedback law 
$u=\beta(x)+u^{\prime}$, with $u^{\prime}$ an auxiliary boundary input, maps (1),

(3) into the target dynamical system

$$
\begin{aligned}
\frac{\partial x}{\partial t}(t, z) & =P_{1} \frac{\partial}{\partial z} \frac{\delta H_{d}}{\delta x}(x(t))(z)+\left(P_{0}-G_{0}\right) \frac{\delta H_{d}}{\delta x}(x(t))(z) \\
u^{\prime}(t) & =W R\left(\begin{array}{c}
\left(\frac{\delta H_{d}}{\delta x}(x(t))\right)(b) \\
\left(\frac{\delta H_{d}}{\delta x}(x(t))\right)(a)
\end{array}\right)
\end{aligned}
$$

with $H_{d}(x)=H(x)+H_{a}(x)$, provided that

$$
\begin{aligned}
P_{1} \frac{\partial}{\partial z} \frac{\delta H_{a}}{\delta x}(x)+\left(P_{0}-G_{0}\right) \frac{\delta H_{a}}{\delta x}(x) & =0 \\
\beta(x)+W R\left(\begin{array}{c}
\left(\frac{\delta H_{a}}{\delta x}(x)\right)(b) \\
\left(\frac{\delta H_{a}}{\delta x}(x)\right)(a)
\end{array}\right) & =0 .
\end{aligned}
$$

Proof: The proof is immediate by comparison of initial and target dynamics. For a geometric interpretation of this result in the distributed parameter scenario, we refer to [22].

Remark 4.1: Equation (26) provides all the possible functions $H_{a}$ that can be employed in the energy-shaping procedure, while (26) gives the associated boundary control action. Furthermore, from (26) it is clear that $\delta H_{a} / \delta x$ is related to the equilibrium states of (1). More precisely, the function $x(t, z):=$ $x_{\star}(z)$ is an equilibrium state of (1) if and only if $x_{\star}:=$ $\mathcal{L}^{-1}\left(\delta H_{a} / \delta x\right)\left(x_{\star}\right)$, with $H_{a}$ such that (26) holds.

Once $H_{d}$ is defined, by Theorem 2.1 a natural choice for the output is

$$
y^{\prime}(t)=\tilde{W} R\left(\begin{array}{c}
\left(\frac{\delta H_{d}}{\delta x}(x(t))\right)(b) \\
\left(\frac{\delta H_{d}}{\delta x}(x(t))\right) \\
(a)
\end{array}\right)
$$

which implies that $(d / d t) H_{d}(x(t)) \leq y^{\prime^{T}}(t) u^{\prime}(t)$. Such new boundary port $\left(u^{\prime}, y^{\prime}\right)$ has now to be terminated over a dissipative element to obtain asymptotic stability of the equilibrium, or just to improve the convergence rate

$$
u^{\prime}(t)=-\Xi y^{\prime}(t), \quad \Xi=\Xi^{T} \geq 0 .
$$

This will be shown in Theorem 5.3.

By the previous remarks it is clear that the additional Hamiltonian $H_{a}$ is constructed in such a way that $\mathcal{L}^{-1}\left(H_{a} / x\right)(x)$ are equilibrium states of (1). Furthermore, since the system has to reach a non-zero state, $H_{d}$ is chosen with a global minimum in this non-zero state. In the following lemma, a construction for $H_{a}$ which achieves this is illustrated. Since, in this paper, the linear case is treated, the focus is on quadratic Hamiltonian functions.

Lemma 4.2: Let $\Phi_{i} \in H^{1}\left(a, b ; \mathbb{R}^{n}\right), \quad i=1, \ldots, n$ be independent solutions of

$$
P_{1} \frac{d \Phi_{i}}{d z}(z)+\left(P_{0}-G_{0}\right) \Phi_{i}(z)=0
$$

and define $\hat{\Phi}(z)=\left(\Phi_{1}(z), \ldots, \Phi_{n}(z)\right)$. Furthermore, let $x_{\star}$ be an equilibrium state of (1), i.e., $\mathcal{L} x_{\star} \in H^{1}\left(a, b ; \mathbb{R}^{n}\right)$ and

$$
P_{1} \frac{\partial\left(\mathcal{L} x_{\star}\right)}{\partial z}(z)+\left(P_{0}-G_{0}\right)\left(\mathcal{L} x_{\star}\right)(z)=0 .
$$

Then

$$
\begin{aligned}
H_{a}(x)= & \frac{1}{2}\left[\int_{a}^{b} \hat{\Phi}^{T}\left(x-x_{\star}\right) d z\right]^{T} \\
& \times Q_{a}\left[\int_{a}^{b} \hat{\Phi}^{T}\left(x-x_{\star}\right) d z\right]-\int_{a}^{b} x_{\star}^{T} \mathcal{L} x d z+\kappa
\end{aligned}
$$

with $Q_{a}=Q_{a}^{T}>0$ and $\kappa \in \mathbb{R}$ some constant, satisfies (26) and $H_{d}=H+H_{a}$ has a global minimum in $x_{\star}$.

Proof: From (32), we have

$$
\frac{\delta H_{a}}{\delta x}(x)=\hat{\Phi} Q_{a}\left[\int_{a}^{b} \hat{\Phi}^{T}\left(x-x_{\star}\right) d z\right]-\mathcal{L} x_{\star}
$$

and so by the definition of $\hat{\Phi}$ and $x_{\star},(26)$ is satisfied. Furthermore, since $H(x)-\int_{a}^{b} x_{\star}^{T} \mathcal{L} x d z$ equals $H\left(x-x_{\star}\right)-H\left(x_{\star}\right)$ the last assertion follows.

\section{Asymptotic StABILITy ANALYSIS}

The aim of this section is now to show that damping injection (29) with $H_{d}=H+H_{a}$ and $H_{a}$ given by (32) asymptotically stabilises (1) in the equilibrium $x_{\star}$. We begin by studying the closed-loop system (25), (28) with (29). Before doing so, we introduce some notation. We define the bounded linear operator $K_{\Phi}: X \rightarrow \mathbb{R}^{n}$ as

$$
K_{\Phi} x=\int_{a}^{b} \hat{\Phi}^{T}(z) x(z) d z
$$

and $\mathcal{L}_{d}$ as

$$
\mathcal{L}_{d}=\mathcal{L}+K_{\Phi}^{*} Q_{a} K_{\Phi}
$$

where $K_{\Phi}^{*}: \mathbb{R}^{n} \rightarrow X$ is the adjoint operator of $K_{\Phi}$. Clearly, $K_{\Phi}^{*}=\hat{\Phi}$, and $\mathcal{L}_{d}$ is a bounded, coercive operator on $L^{2}\left(a, b ; \mathbb{R}^{n}\right)$. Furthermore, $H_{a}$ being given by (32) we find

$$
H_{d}(x)=\frac{1}{2}\left\langle\left( x-x_{\star}\left|\mathcal{L}_{d}\left(x-x_{\star}\right)\right\rangle_{L^{2}}+H_{d}\left(x_{\star}\right) .\right.\right.
$$

Proposition 5.1: The closed-loop system (25), (28) with (29) in which $H_{a}$ is defined by (32) admits a unique solution. Furthermore, the mapping from the initial error state at time $t=0, x_{0}-x_{\star}$ to the error state at time $t, x(t)-x_{\star}$ defines a contraction semigroup in the norm $(1 / 2)\left\langle\left(x-x_{\star}\right)\right| \mathcal{L}_{d}(x-$ $\left.\left.x_{\star}\right)\right\rangle_{L^{2}}$.

Proof: We begin by defining $\tilde{x}$ as $x-x_{\star}$, then by (35) we have that $\left(\delta H_{d} / \delta x\right)(x)=\mathcal{L}_{d} \tilde{x}$. Since $x_{\star}$ is independent of 
$t$, we see that the closed-loop system (25), (28) with (29) can be written as

$$
\begin{aligned}
\frac{\partial \tilde{x}}{\partial t}(t, z) & =P_{1} \frac{\left(\partial \mathcal{L}_{d} \tilde{x}\right)}{\partial z}(t, z)+\left(P_{0}-G_{0}\right)\left(\mathcal{L}_{d} \tilde{x}\right)(t, z) \\
0 & =[W+\Xi \tilde{W}] R\left(\begin{array}{c}
\left(\mathcal{L}_{d} \tilde{x}\right)(t, b) \\
\left(\mathcal{L}_{d} \tilde{x}\right)(t, a)
\end{array}\right) .
\end{aligned}
$$

By pre- and post-multiplication of (6) by $\left(\begin{array}{lll}I & \Xi\end{array}\right)$ and $\left(\begin{array}{ll}I \quad \Xi^{T}\end{array}\right)$, respectively, we obtain:

$$
[W+\Xi \tilde{W}] \Sigma[W+\Xi \tilde{W}]^{T}=2 \Xi \geq 0
$$

and then from Lemma 5.4 in [8] it follows that the semigroup associated to (36) is a contraction semigroup with respect to the norm $(1 / 2)\left\langle\left(x-x_{\star}\right) \mid \mathcal{L}_{d}\left(x-x_{\star}\right)\right\rangle_{L^{2}}$. Furthermore, since $\tilde{x}$ and $x$ only differ by $x_{\star}$ is clear that the closed-loop system (25), (28) with (29) admits a unique mild solution for all initial conditions.

Proposition 5.2: The operator $\mathcal{J}_{d}$ defined as

$$
\mathcal{J}_{d} x:=P_{1} \frac{\partial\left(\mathcal{L}_{d} x\right)}{\partial z}+\left(P_{0}-G_{0}\right)\left(\mathcal{L}_{d} x\right)
$$

with domain

$$
\begin{array}{r}
D\left(\mathcal{J}_{d}\right)=\left\{x \in L^{2}\left(a, b ; \mathbb{C}^{n}\right) \mid \mathcal{L}_{d} x \in H^{1}\left(a, b ; \mathbb{C}^{n}\right)\right. \\
\text { and } \left.0=[W+\Xi \tilde{W}] R\left(\begin{array}{c}
\left(\mathcal{L}_{d x}\right)(b) \\
\left(\mathcal{L}_{d x}\right)(a)
\end{array}\right)\right\}
\end{array}
$$

is the infinitesimal generator of a contraction semigroup and has a compact resolvent.

Proof: From [8, Lemma 5.4], of which Theorem 2.1 is a particular case, it follows that $\mathcal{J}_{d}$ generates a contraction semigroup since $\mathcal{L}_{d}$ is a bounded, coercive operator on $L^{2}\left(a, b ; \mathbb{R}^{n}\right)$. The compactness of the resolvent is derived from [25, Theorem 2.28, pg. 50] because, as before, $\mathcal{L}_{d}$ is a bounded and coercive operator.

The main result is an application of the Arendt-BattyLyubich-Vũ Theorem, see e.g., [29, Theorem 3.26, p. 130].

Theorem 5.3 (Asymptotic Stability): Consider the linear, infinite dimensional, port-Hamiltonian system (1) and the equilibrium state $x_{\star}$ satisfying (31). Then, the control action $u=$ $\beta(x)+u^{\prime}$ with $\beta$ defined in (27), $H_{a}$ chosen as in (32), and with $u^{\prime}$ defined in (29) with $\Xi>0$, makes $x_{\star}$ asymptotically stable.

Proof: Using the previous notation, it is clear that the assertion in the theorem is equivalent to the assertion that the origin is asymptotically stable for the PDE (36). To this PDE, we associate the infinitesimal generator $\mathcal{J}_{d}$ defined by (37) and with domain (38). Since $\mathcal{J}_{d}$ has compact resolvent and generates a contraction semigroup, the semigroup is asymptotically stable if and only if there are no eigenvalues on the imaginary axis, see [29, Theorem 3.26]. In this respect, assume that $\jmath \omega$ is an eigenvalue, i.e., there exists a nonzero $x \in D\left(\mathcal{J}_{d}\right)$ such that

$$
\jmath \omega x=\mathcal{J}_{d} x .
$$

Using the definition of $\mathcal{J}_{d}$ and integration by parts, we see that

$$
\begin{aligned}
0= & \operatorname{Re}\left(\left\langle\mathcal{L}_{d} x \mid \jmath \omega x\right\rangle\right) \\
= & \frac{1}{2}\left(\mathcal{L}_{d} x\right)^{*}(b) P_{1}\left(\mathcal{L}_{d} x\right)(b)-\frac{1}{2}\left(\mathcal{L}_{d} x\right)^{*}(a) P_{1}\left(\mathcal{L}_{d} x\right)(a) \\
& \quad-\left\langle\mathcal{L}_{d} x \mid G_{0} \mathcal{L}_{d} x\right\rangle \\
= & \frac{1}{2}\left(y^{\prime}\right)^{*} u^{\prime}+\frac{1}{2}\left(u^{\prime}\right)^{*} y^{\prime}-\left\langle\mathcal{L}_{d} x \mid G_{0} \mathcal{L}_{d} x\right\rangle
\end{aligned}
$$

where we have introduced

$$
u^{\prime}=W R\left(\begin{array}{c}
\left(\mathcal{L}_{d} x\right)(b) \\
\left(\mathcal{L}_{d} x\right)(a)
\end{array}\right) \quad y^{\prime}=\tilde{W} R\left(\begin{array}{c}
\left(\mathcal{L}_{d} x\right)(b) \\
\left(\mathcal{L}_{d} x\right)(a)
\end{array}\right)
$$

and used (6). Hence, the boundary condition gives

$$
0=-\left(y^{\prime}\right)^{*} \Xi y^{\prime}-\left\langle\mathcal{L}_{d} x \mid G_{0} \mathcal{L}_{d} x\right\rangle
$$

Since $\Xi>0$ we see that $y^{\prime}=0$ and thus $u^{\prime}=0$. Furthermore,

$$
G_{0} \mathcal{L}_{d} x=0 .
$$

Using the fact that $\left(\begin{array}{c}W \\ \tilde{W}\end{array}\right)$ is invertible, $y^{\prime}=u^{\prime}=0$ implies that $\left(\mathcal{L}_{d} x\right)(a)=\left(\mathcal{L}_{d} x\right)(b)=0$. Let now consider two cases:

- If $\omega=0$, then (39) and (41) imply that the function $q:=$ $\mathcal{L}_{d} x$ satisfies the first order ordinary differential equation $P_{1} \frac{\partial q}{\partial z}+P_{0} q=0$. However, since $q(b)=q(a)=0$ this is only possible when $q \equiv 0$. Thus zero is not an eigenvalue.

- For $\omega \neq 0$, we introduce $\xi=\int_{a}^{b} \hat{\Phi}^{T}(z) x(z) d z$. We have that

$$
\begin{aligned}
\jmath \omega \xi & =\int_{a}^{b} \hat{\Phi}^{T}(z) \jmath \omega x(z) d z \\
& =\int_{a}^{b} \hat{\Phi}^{T}(z)\left(\mathcal{J}_{d} x\right)(z) d z \\
& =\hat{\Phi}^{T}(b) P_{1}\left(\mathcal{L}_{d} x\right)(b)-\hat{\Phi}^{T}(a) P_{1}\left(\mathcal{L}_{d} x\right)(a)
\end{aligned}
$$

where we have used integration by parts (30) and (41). Since $\left(\mathcal{L}_{d} x\right)(a)=\left(\mathcal{L}_{d} x\right)(b)=0$, we have proved that $\xi=0$. Combining this with (33) and (34) we see that

$$
\mathcal{L}_{d} x=\mathcal{L} x+K_{\Phi}^{*} Q_{a} \xi=\mathcal{L} x
$$

Using this and the definition of $\mathcal{J}_{d}$, we have that $x$ satisfies the first order ordinary differential equation

$$
\jmath \omega x=P_{1} \frac{\partial(\mathcal{L} x)}{\partial z}+P_{0}(\mathcal{L} x)
$$

Since $\mathcal{L}$ is Lipschitz continuous, bounded from above and away from zero, so is its inverse. Due to the Cauchy-Lipschitz theorem on existence and uniqueness of solutions to ordinary differential equations with given initial conditions, and combining this fact with $\left(\mathcal{L}_{d} x\right)(a)=(\mathcal{L} x)(a)=0$, we conclude that $x=0$. Hence there are no eigenvalues on the imaginary axis and the closed-loop error system is asymptotically stable. 
Remark 5.1: If in (32) it is assumed that the functions $\Phi_{i}$ solutions of (30) are such that

$$
0=W R\left(\begin{array}{c}
\Phi_{i}(b) \\
\Phi_{i}(a)
\end{array}\right), \quad i=1, \ldots, n
$$

then the energy-shaping state feedback law $\beta$ defined in (27) reduces to a constant, namely

$$
\beta(x)=W R\left(\begin{array}{c}
\hat{\Phi}(b) \phi_{\star} \\
\hat{\Phi}(a) \phi_{\star}
\end{array}\right)
$$

which are the boundary conditions associated to the equilibrium $(\mathcal{L} x)_{\star}$. Then, the effect of the damping injection contribution (29) is to dissipate the total energy until the new minimum is reached. A simple application of [30] shows that the equilibrium is uniformly exponentially stable. Since there are no constraints on the boundary conditions on the function $\Phi_{i}$ solution of (30), a parametrization of all the possible energyshaping control actions is provided in the linear case. Different choices lead to different performances in closed-loop.

Remark 5.2: With the methodology discussed in the previous section in mind, provided that $H_{C}(x) \equiv H_{a}(x)$ and $H_{\mathrm{cl}}(x) \equiv$ $H_{d}(x)$, we see that the control by interconnection and energy shaping via Casimir generation is a particular case of this one. In fact, since the Casimir functions have to satisfy (17) and (20), it is immediate that

$$
P_{1} \frac{d \Psi}{d z}(z)+\left(P_{0}-G_{0}\right) \Psi(z)=0
$$

and that

$$
\frac{\delta H_{C}}{\delta x}(x(t, z))=\left.\Psi(z) \frac{\partial H_{C}}{\partial x_{C}}\right|_{x_{C}=\int_{a}^{b} \Psi^{T}(z) x(t, z) d z} .
$$

Furthermore, condition (27) is a consequence of the definition of $u^{\prime}$ in (23). In addition, if for simplicity the finite dimensional boundary controller (8) is chosen without the feedthrough term, i.e., if $M_{C}=S_{C}=0$, then in the second relation in (23) we have that $W^{\prime}=W$. Since $u=\beta(x)+u^{\prime}$, from (3) we have that

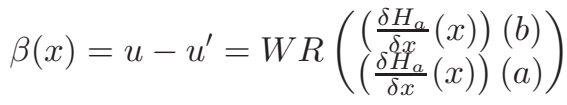

which is exactly (27). Equivalently, we can say that in the lossless case for any energy-shaping control action $\beta(x)$ it is possible to determine a control system (8) that is able, if properly initialised, to generate the control action $\beta(x)$ itself.

\section{EXAMPLE: THE LONGITUDINAL VIBRATION OF A BEAM}

\section{A. Port Hamiltonian Modelling}

In this section, we consider the example of a bar of size $L$ subject to longitudinal (axial) vibration. The beam motion results from an extension/compression deformation along its longitudinal direction $z \in[0, L]$. In the following, we shall denote the section of the beam by $S(z)$, the longitudinal displacement of a section of the beam from the unstressed configuration by $\varphi(t, z)$, and its velocity by $v=(\partial \varphi / \partial t)(t, z)$. In case of longitudinal motion, the deformation of the beam $\varepsilon(t, z)$ is related to the displacement by:

$$
\varepsilon(t, z)=\frac{\partial \varphi}{\partial z}(t, z)
$$

The material's deformation behavior is considered to be linear (Hooke's law), which means that the axial mechanical constraint $\sigma(t, z)$, defined as the extension/traction force divided by section $S(z)$, is proportional to the axial deformation $\varepsilon(t, z)$ through the Young elasticity modulus $E$, i.e., $\sigma(t, z)=$ $E \varepsilon(t, z)$. Applying the second Newton's law to an infinitesimal piece of beam (taking internal friction into account) leads to the PDE equation

$$
\rho S(z) \frac{\partial^{2} \varphi}{\partial t^{2}}(t, z)=\frac{\partial}{\partial z}\left[E S(z) \frac{\partial \varphi}{\partial z}(t, z)\right]-D \frac{\partial \varphi}{\partial t}(t, z)
$$

where $\rho$ is the mass density, and $D \geq 0$ is the internal friction coefficient. By considering as energy variables the deformation $\varepsilon(t, z)$ and the linear momentum density $p(t, z)=$ $\rho S(z) v(t, z)$, the total energy of the system can be written as the sum of the kinetic energy and the potential energy of the elastic deformation, i.e.,:

$$
H(p(t, z), \varepsilon(t, z))=\frac{1}{2} \int_{0}^{L}\left[\frac{p^{2}(t, z)}{\rho S(z)}+E S(z) \varepsilon^{2}(t, z)\right] d z
$$

leading to the definition of the co-energy variables

$$
\begin{aligned}
\sigma_{S}(t, z) & =\frac{\delta H}{\delta \varepsilon}(\varepsilon(t, z))=\operatorname{ES}(z) \varepsilon(t, z)=S(z) \sigma(t, z) \\
v(t, z) & =\frac{\delta H}{\delta p}(p(t, z))=\frac{p(t, z)}{\rho S(z)}=\frac{\partial \varphi}{\partial t}(t, z)
\end{aligned}
$$

which are the elastic force acting on the cross-section, and its velocity, respectively. The port-Hamiltonian formulation of the system is then

$$
\frac{\partial}{\partial t}\left(\begin{array}{l}
\varepsilon(t, z) \\
p(t, z)
\end{array}\right)=\left(\begin{array}{cc}
0 & \frac{\partial}{\partial z} \\
\frac{\partial}{\partial z} & -D
\end{array}\right)\left(\begin{array}{cc}
E S(z) & 0 \\
0 & \frac{1}{\rho S(z)}
\end{array}\right)\left(\begin{array}{l}
\varepsilon(t, z) \\
p(t, z)
\end{array}\right)
$$

which is in the form (1), with $P_{0}=0$, and

$$
\begin{aligned}
P_{1} & =\left(\begin{array}{ll}
0 & 1 \\
1 & 0
\end{array}\right), \quad G_{0}=\left(\begin{array}{cc}
0 & 0 \\
0 & D
\end{array}\right) \\
\mathcal{L}(z) & =\left(\begin{array}{cc}
E S(z) & 0 \\
0 & \frac{1}{\rho S(z)}
\end{array}\right) .
\end{aligned}
$$

The boundary port variables (2) are

$$
\left(\begin{array}{c}
f_{\partial} \\
e_{\partial}
\end{array}\right)=\frac{1}{\sqrt{2}}\left(\begin{array}{c}
v(L)-v(0) \\
\sigma_{S}(L)-\sigma_{S}(0) \\
\sigma_{S}(L)+\sigma_{S}(0) \\
v(L)+v(0)
\end{array}\right)
$$

The boundary input and output are selected as

$$
u(t)=\left(\begin{array}{c}
v(t, 0) \\
\sigma_{S}(t, L)
\end{array}\right), \quad y(t)=\left(\begin{array}{c}
-\sigma_{S}(t, 0) \\
v(t, L)
\end{array}\right)
$$


which can be derived from (3) and (4) thanks to the following choice for $W$ and $\tilde{W}$ :

$$
W=\frac{1}{\sqrt{2}}\left(\begin{array}{cccc}
-1 & 0 & 0 & 1 \\
0 & 1 & 1 & 0
\end{array}\right), \quad \tilde{W}=\frac{1}{\sqrt{2}}\left(\begin{array}{cccc}
0 & 1 & -1 & 0 \\
1 & 0 & 0 & 1
\end{array}\right) .
$$

The energy balance associated to this choice of input and output is then given by

$$
\frac{d H}{d t}(t)=-\int_{0}^{L} D v^{2}(t, z) d z+y^{T}(t) u(t) \leq y^{T}(t) u(t) .
$$

\section{B. Lossless Case}

At first, we assume that $D=0$, and we consider the fully actuated case, i.e., the controller acts on both sides of the beam, and a state feedback of the form $u(t)=\beta(\varepsilon, p)+u^{\prime}$. The aim of the state feedback is to shape, at least partially, the closedloop energy function. The stability is insured by an additional dissipation term on the new input/output. From Lemma 4.2, the class of function $H_{a}$ that can be employed in the energyshaping design procedure are in the form

$$
H_{a}(\varepsilon, p)=\hat{H}_{a}\left(\xi_{1}(\varepsilon, p), \xi_{2}(\varepsilon, p)\right)
$$

with

$$
\begin{aligned}
\xi_{1}(\varepsilon(t, \cdot)) & =\int_{0}^{L} \varepsilon(t, z) d z \\
\xi_{2}(p(t, \cdot)) & =\int_{0}^{L} p(t, z) d z
\end{aligned}
$$

and $\hat{H}_{a}$ can be freely chosen. A closed-loop system with Hamiltonian $H_{d}(\varepsilon, p)=H(\varepsilon, p)+H_{a}(\varepsilon, p)$ with a minimum in $(0,0)$ is obtained by selecting $\hat{H}_{a}$ as

$$
\hat{H}_{a}\left(\xi_{1}, \xi_{2}\right)=\frac{1}{2} \Xi_{1} \xi_{1}^{2}+\frac{1}{2} \Xi_{2} \xi_{2}^{2}
$$

where $\Xi_{1}, \Xi_{2}$ are two positive gains. From (27), this leads to the state feedback:

$$
\beta(\varepsilon, p)=-\left(\begin{array}{l}
\Xi_{1} \xi_{2}(p) \\
\Xi_{1} \xi_{1}(\varepsilon)
\end{array}\right)=-\left(\begin{array}{cc}
\Xi_{2} & 0 \\
0 & \Xi_{1}
\end{array}\right)\left(\begin{array}{l}
\int_{0}^{L} p d z \\
\int_{0}^{L} \varepsilon d z
\end{array}\right)
$$

and the desired closed-loop energy function:

$$
\begin{aligned}
H_{d}(\varepsilon, p)=\frac{1}{2} \int_{0}^{L}\left[\frac{p^{2}}{\rho S(z)}+E S(z) \varepsilon^{2}\right] d z \\
+\frac{1}{2} \Xi_{1}\left(\int_{0}^{L} \varepsilon d z\right)^{2}+\frac{1}{2} \Xi_{2}\left(\int_{0}^{L} p d z\right)^{2} .
\end{aligned}
$$

The resulting closed-loop system is impedance passive with respect to the new input/output port $\left(u^{\prime}, y^{\prime}\right)$ defined by (25) and (28). Moreover, from (46) and (47), we see the energy function can be (partially) shaped in the $\varepsilon$ and $p$ coordinates by adequately choosing the gains $\Xi_{1}$ and $\Xi_{2}$. The asymtotic stability is obtained by interconnecting a dissipative element at the input/output port $\left(u^{\prime}, y^{\prime}\right)$, as in (29). The achievable performances of energy shaping plus damping injection control strategy are illustrated in Section VI-D.

Remark 6.1: A similar result could have been obtained by using the energy-Casimir method. For that purpose, let us consider the system (8) with $n_{C}=2, R_{C}=P_{C}=M_{C}=S_{C}=0$, $G_{C}=I$ and $J_{C}$ to be assigned later on. By following the energy-Casimir method discussed in Section III, it is quite easy to check that Casimir functions are not present in closed-loop if $J_{C}=0$. With this choice, the boundary controller (8) consists of two separate systems, each required to provide a constant power flow in steady state: they are not energy-balancing controllers. So, it is necessary to couple these regulators and allow for an internal power flow at the controller side. This can be achieved by choosing

$$
J_{C}=\left(\begin{array}{cc}
0 & I \\
-I & 0
\end{array}\right)
$$

which implies that the closed-loop system is characterized by the following Casimir functions:

$$
\begin{aligned}
& C_{1}\left(\xi_{1}(t), \varepsilon(t, \cdot)\right)=\xi_{1}(t)-\int_{0}^{L} \varepsilon(t, z) d z \\
& C_{2}\left(\xi_{2}(t), p(t, \cdot)\right)=\xi_{2}(t)-\int_{0}^{L} p(t, z) d z .
\end{aligned}
$$

Note the similarities with (45), as expected. The controller Hamiltonian can then be chosen as in (46).

One can check that the closed-loop system is lossless, so only simple stability has been achieved. However, asymptotic stability can be obtained by damping injection at the boundary, as discussed in Section IV. More precisely, asymptotic stability follows immediately from Theorem 5.3

\section{System With Internal Friction}

Due to internal dissipation, i.e., when $D \neq 0$, the energyCasimir method briefly discussed at the end of the previous subsection (using a dynamic controller, and reduction) cannot be applied as the dissipation obstacle does not allow to compute invariant Casimir function in the $p$ coordinate. It is then necessary to rely on the energy-shaping methodology presented in Section IV. The PDE (26) provides the admissible functions $H_{a}$, and (27) the associated boundary control action.

With Lemma 4.2 in mind, the admissible $H_{a}$ takes again the form (41), with now

$$
\xi_{1}(\varepsilon(t, \cdot))=\int_{0}^{L} \varepsilon(t, z) d z
$$

$$
\xi_{1}(\varepsilon(t, \cdot), p(t, \cdot))=\int_{0}^{L}[D(L-z) \varepsilon(t, z)+p(t, z)] d z .
$$




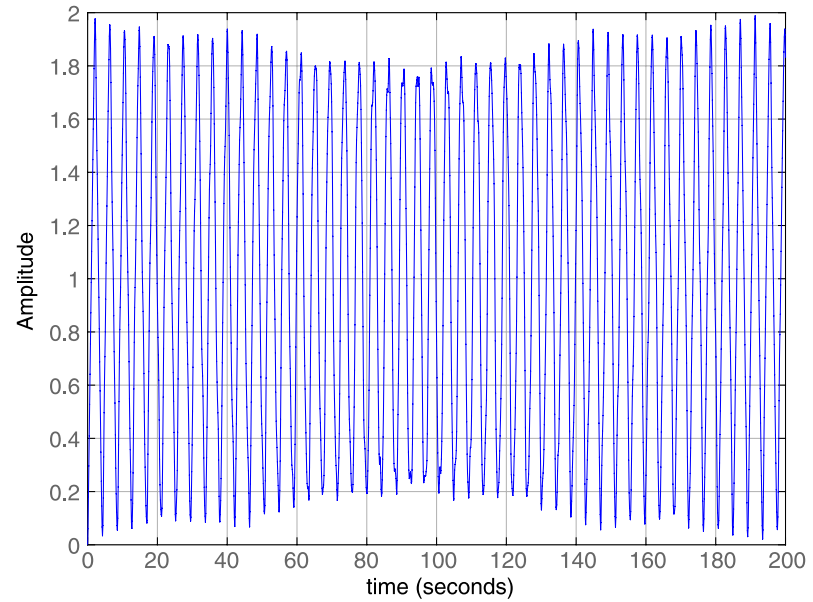

Fig. 1. Open-loop step response.

Note that the solution proposed in [35] is just a particular case of the one presented here. Finally, $H_{a}$ can be selected e.g., as in (46) and, thanks to Theorem 5.3, asymptotic stability is obtained via damping injection (29) on the new control port $\left(u^{\prime}, y^{\prime}\right)$ defined in (25) and (28) in the general case.

\section{Achievable Closed-Loop Performances}

In order to illustrate the achievable performances with the energy-shaping methods proposed in this paper, we consider the aforementioned beam (with $D=0$ ) clamped at one side and controlled at the other side, i.e.,

$$
\begin{aligned}
& u(t)=\left(\begin{array}{c}
v(t, 0) \\
\sigma_{S}(t, L)
\end{array}\right)=\left(\begin{array}{c}
0 \\
\bar{u}(t)
\end{array}\right) \\
& y(t)=\left(\begin{array}{c}
-\sigma_{S}(t, 0) \\
v(t, L)
\end{array}\right)=\left(\begin{array}{c}
\tilde{y}(t) \\
\bar{y}(t)
\end{array}\right)
\end{aligned}
$$

where $u$ and $y$ are defined as in (43), $\bar{u}$ is the actual control input, namely the applied force in $z=L$, and $\bar{y}$ the associated dual output, the velocity in $z=L$.

1) Open-Loop Response: For simulation purpose, we consider a finite dimensional approximation of the system with normalized parameters (all set equal to one). In particular, the spatial discretisation technique for distributed port-Hamiltonian systems presented in [36] has been employed. The result is a finite volume approximation in port-Hamiltonian form. Fig. 1 shows the evolution of the position of the end of the beam when a (normalized) force step is applied at the same point. One can note the undamped oscillations occurring at the different frequencies.

2) Dissipative Boundary Feedback: At first, a dissipative boundary feedback in the form

$$
\bar{u}(t)=-\alpha \bar{y}(t), \quad \alpha>0
$$

is implemented. Fig. 2 clearly shows that the oscillations can be damped by increasing the values of $\alpha$. As long as the system is damped, the raising time increases, but at the same time, the settling time decreases to $2.5 \mathrm{~s}$ until $\alpha$ is tuned in such a way that the system does not present any oscillations. This

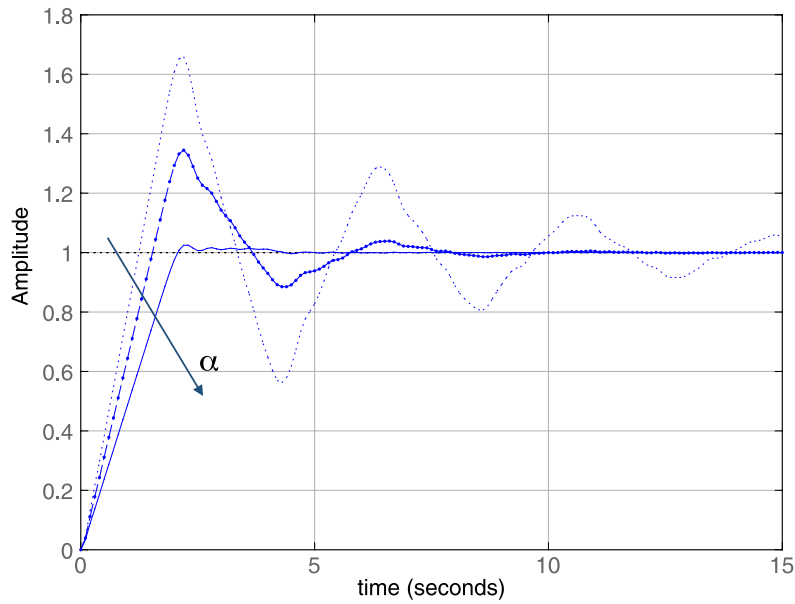

Fig. 2. Different step responses for increasing values of $\alpha>0$ (with dot $\alpha=0.2$, dashed $\alpha=0.5$, and solid line $\alpha=1$ symbols, accordingly).

happens when $\alpha=1$, i.e., when the dissipative gain matches the mechanical impedance of the beam. For larger values of $\alpha$, the system is over-damped, and the settling time increases again.

3) Energy Shaping: We consider now the energy-shaping method presented in Section IV. Since one of the extremities of the beam is clamped, $H_{a}$ is looked for under the form $H_{a}(\varepsilon)=$ $\hat{H}_{a}\left(\xi_{1}(\varepsilon)\right)$. By applying Lemma 4.2, the admissible $H_{a}$ are of the form

$$
H_{a}(\varepsilon(t, \cdot))=\frac{\Xi}{2}\left(\int_{0}^{L} \varepsilon(t, z) d z\right)^{2}=\frac{\Xi}{2}[\varphi(t, L)-\varphi(t, 0)]^{2}
$$

with $\Xi>0$, in which the geometric constraint (42) has been taken into account. The corresponding state feedback is

$$
\beta(\varphi)=-\Xi_{1}[\varphi(t, L)-\varphi(t, 0)]
$$

which is equivalent to an additional boundary stiffness, i.e., to a proportional control action. Asymptotic stability is achieved thanksto a dissipative feedback gain $\alpha$, and the final control law is of the form

$$
\begin{aligned}
u=\beta(\varphi)-\alpha \bar{y} & =-\Xi[\varphi(t, L)-\varphi(t, 0)]-\alpha v(t, L) \\
& =-\Xi \varphi(t, L)-\alpha v(t, L)
\end{aligned}
$$

in which it is assumed that $\varphi(t, 0)=0$ because the beam is clamped in $z=0$. Note that this is a classical PD control law, in which the proportional gain is related to energy-shaping, while the derivative one to damping injection. Fig. 3 shows how $\Xi$ allows to improve the settling time, and this effect combined with the damping injection gain $\alpha$ allows to improve drastically the transient response.

\section{CONCLUSIONS AND FUTURE WORK}

The motivating idea of the paper has been the development of a general synthesis methodology of boundary control laws 


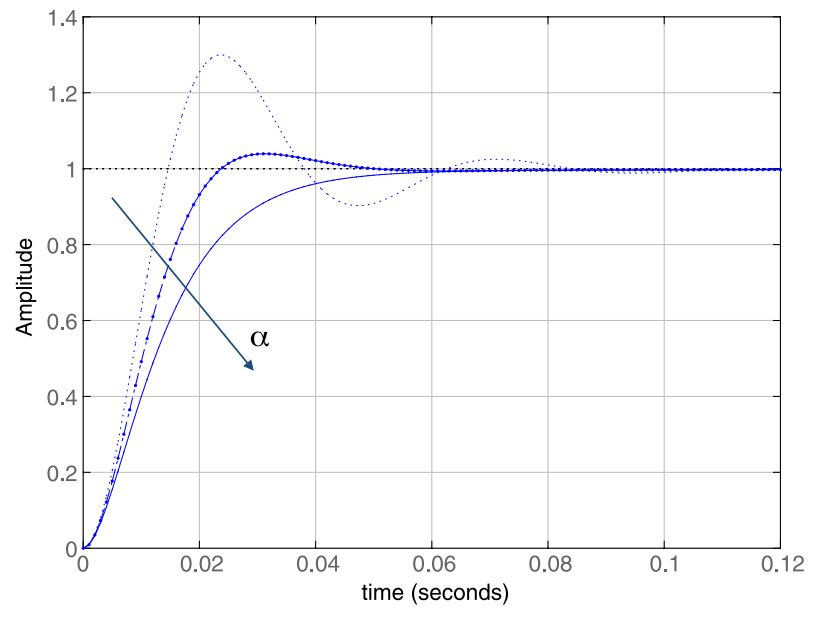

Fig. 3. Closed-loop response in case of energy-shaping plus damping injection control law with fixed $\Xi=200$, and increasing values of $\alpha \geq 0$ (with dot $\alpha=10$, dashed $\alpha=20$, and solid line $\alpha=30$ symbols, accordingly).

for linear, distributed port-Hamiltonian systems on a onedimensional spatial domain. As in the lumped parameter case, the feedback law is determined in such a way that its effect on the system is to shape the energy function, and to modify the dissipative structure. Thanks to energy-shaping, simple stability of the desired equilibrium is achieved, while damping injection assures asymptotic convergence of the trajectories. For any infinite-dimensional system existence and uniqueness of solutions is not guaranteed beforehand. Therefore, we started with the energy-Casimir method to design our control action that leads to a (formally) passive dynamical system. Using this structure it is much easier to prove that the set of PDEs and ODEs associated with the dynamics of the closed-loop system has a unique solution. This property holds also when the control action is not provided by a dynamic controller, but by an equivalent state feedback law.

Since the class of stabilizing controllers that the energyCasimir method can provide is quite limited because of the dissipation obstacle, the problem of determining a feedback law able to shape the Hamiltonian in a proper manner has been tackled by determining the control action that maps the open-loop system into a new one, with the same geometric structure, but with a different Hamiltonian. Since the control action shares the main properties of the feedback law obtained via the energy-Casimir method, it is possible to verify that also in this case the closed-loop system is well-posed, and defines a new boundary control system. The resulting control law is proved to asymptotically stabilize the system.

The proposed methodology has been developed for linear systems with one-dimensional domain. The extensions to distributed port-Hamiltonian systems on a 2-D or 3-D spatial domain and to non linear distributed port-Hamiltonian systems are our main future research topics. Concerning the later one, all the geometric considerations that have been used in this paper remain valid as the port-Hamiltonian framework is intrinsically devoted to non linear systems, but the analysis of the existence of solution and of the stability proof remain difficult and open problems.

\section{APPENDIX \\ Proofs of the Results of Section III}

\section{A. Proof of Proposition 3.1}

By using the compact notation introduced in (10), and with Definition 3.1 in mind, $(d C / d t)=0$ along all classical solutions if and only if for all $\left(\mathcal{L} x, x_{C}\right) \in H^{1}\left(a, b ; \mathbb{R}^{n}\right) \times \mathbb{R}^{n_{C}}$ there holds

$$
\begin{aligned}
\mathcal{B}^{\prime}\left(\begin{array}{c}
x \\
x_{C}
\end{array}\right)= & 0 \\
0= & \Gamma^{T}\left[A_{C} x_{C}+B_{c} u_{C}\right]+ \\
& +\int_{a}^{b} \Psi^{T}\left[P_{1} \frac{\partial(\mathcal{L} x)}{\partial z}+\left(P_{0}-G_{0}\right)(\mathcal{L} x)\right] d z
\end{aligned}
$$

Since (49) holds for all $\mathcal{L} x \in H^{1}\left(a, b ; \mathbb{R}^{n}\right)$, it implies that $\Psi \in$ $H^{1}\left(a, b ; \mathbb{R}^{n}\right)$. By integrating by parts, we find

$$
\begin{aligned}
0= & \Gamma^{T}\left[A_{C} x_{C}+B_{C} u_{C}\right] \\
& +\int_{a}^{b}\left[-\left(\frac{d \Psi}{d z}\right)^{T} P_{1}+\Psi^{T}\left(P_{0}-G_{0}\right)\right](\mathcal{L} x) d z \\
& +\left(\begin{array}{l}
\Psi(b) \\
\Psi(a)
\end{array}\right)^{T}\left(\begin{array}{cc}
P_{1} & 0 \\
0 & -P_{1}
\end{array}\right)\left(\begin{array}{l}
(\mathcal{L} x)(b) \\
(\mathcal{L} x)(a)
\end{array}\right)
\end{aligned}
$$

By the definition of a Casimir, the above has to hold independently of $\mathcal{L}$ and $Q_{C}$. The integral term vanishes if and only if $\Psi$ satisfies (17), where we used the properties of $P_{1}, P_{0}$ and $G_{0}$. Next, we concentrate on the equation (50) without the integral term. Using (3), (4), and (11) with $u^{\prime}=0$, we have that

$$
\begin{aligned}
& W\left(\begin{array}{l}
f_{\partial} \\
e_{\partial}
\end{array}\right)=u=-y_{C}=-C_{C} x_{C}-D_{C} u_{C} \\
& \tilde{W}\left(\begin{array}{l}
f_{\partial} \\
e_{\partial}
\end{array}\right)=y=u_{C} .
\end{aligned}
$$

Thanks to (6) and the definition of $\Sigma$, we see that the inverse of $\left(\begin{array}{c}W \\ \tilde{W}\end{array}\right)$ equals $\Sigma\left(W^{T} \quad \tilde{W}^{T} \Sigma\right.$. Thus

$$
\left(\begin{array}{l}
f_{\partial} \\
e_{\partial}
\end{array}\right)=\Sigma\left(\begin{array}{ll}
W^{T} & \tilde{W}^{T}
\end{array}\right)\left(\begin{array}{c}
u_{C} \\
-C_{C} x_{C}-D_{C} u_{C}
\end{array}\right) .
$$

By using the above relation, the equality

$$
\left(\begin{array}{cc}
P_{1} & 0 \\
0 & -P_{1}
\end{array}\right)=R^{T} \Sigma R
$$

and (2), we see that (50) becomes

$$
\begin{aligned}
0=\Gamma^{T} & {\left[A_{C} x_{C}+B_{C} u_{C}\right] } \\
& +\left(\begin{array}{l}
\Psi(b) \\
\Psi(a)
\end{array}\right)^{T} R^{T}\left(\begin{array}{ll}
W^{T} & \tilde{W}^{T}
\end{array}\right)\left(\begin{array}{c}
u_{C} \\
-C_{C} x_{C}-D_{C} u_{C}
\end{array}\right)
\end{aligned}
$$


or equivalently by using (10) and $u_{C}=y$

$$
\begin{aligned}
0= & {\left[\Gamma^{T}\left(J_{C}-R_{C}\right)-\left(\begin{array}{l}
\Psi(b) \\
\Psi(a)
\end{array}\right)^{T} R^{T} \tilde{W}^{T}\left(G_{C}+P_{C}\right)^{T}\right] Q_{C} x_{C} } \\
& +\left[\Gamma^{T}\left(G_{C}-P_{C}\right)+\left(\begin{array}{l}
\Psi(b) \\
\Psi(a)
\end{array}\right)^{T} R^{T}\left(W^{T}-\tilde{W}^{T}\left(M_{C}+S_{C}\right)\right)\right] y .
\end{aligned}
$$

From the definition of $y$ in (4) and the skew symmetry of $J_{C}$ and $M_{C}$, this expression becomes independent of $Q_{C}$ and $\mathcal{L}(\cdot)$ if and only if (18) and (19) hold. Since the classical solution are dense, the assertion follows.

\section{B. Proof of Proposition 3.2}

Let us consider the matrices $W$ and $\tilde{W}$ introduced in Theorem 2.1, and satisfying (6). Then, the skew-symmetric and symmetric parts of $\tilde{W}^{T} W$ are given by

$$
\begin{aligned}
\tilde{J}= & \frac{1}{2}\left[\tilde{W}^{T} W-W^{T} \tilde{W}\right] \\
& \frac{1}{2}\left[\tilde{W}^{T} W+W^{T} \tilde{W}\right]=\frac{1}{2} \Sigma
\end{aligned}
$$

respectively, where (6) was used in the last relation. We can then write that

$$
\tilde{W}^{T} W=\tilde{J}+\frac{1}{2} \Sigma .
$$

Now, since

$$
\begin{aligned}
& \left(\begin{array}{c}
\hat{\Psi}(b) \\
\hat{\Psi}(a)
\end{array}\right)^{T}\left(\begin{array}{cc}
P_{1} & 0 \\
0 & -P_{1}
\end{array}\right)\left(\begin{array}{c}
\hat{\Psi}(b) \\
\hat{\Psi}(a)
\end{array}\right)= \\
& \quad=\int_{a}^{b} \frac{d \hat{\Psi}^{T}}{d z}(z) P_{1} \hat{\Psi}(z)+\hat{\Psi}^{T}(z) P_{1} \frac{d \hat{\Psi}}{d z}(z) d z
\end{aligned}
$$

we find by using (17), the symmetry of $P_{1}, G_{0}$, and the skewsymmetry of $P_{0}$ that

$$
\begin{gathered}
\left(\begin{array}{c}
\hat{\Psi}(b) \\
\hat{\Psi}(a)
\end{array}\right)^{T}\left(\begin{array}{cc}
P_{1} & 0 \\
0 & -P_{1}
\end{array}\right)\left(\begin{array}{l}
\hat{\Psi}(b) \\
\hat{\Psi}(a)
\end{array}\right)= \\
=-2 \int_{a}^{b} \hat{\Psi}^{T}(z) G_{0} \hat{\Psi}(z) d z .
\end{gathered}
$$

By eliminating $G_{C}$ in (18) and (19), we have that

$$
\begin{aligned}
0= & \hat{\Gamma}^{T}\left(J_{C}+R_{C}\right) \hat{\Gamma}+2 \hat{\Gamma}^{T} P_{C} \tilde{W} R\left(\begin{array}{c}
\hat{\Psi}(b) \\
\hat{\Psi}(a)
\end{array}\right) \\
& +\left(\begin{array}{c}
\hat{\Psi}(b) \\
\hat{\Psi}(a)
\end{array}\right)^{T} R^{T} \tilde{W}^{T}\left(M_{C}+S_{C}\right) \tilde{W} R\left(\begin{array}{c}
\hat{\Psi}(b) \\
\hat{\Psi}(a)
\end{array}\right) \\
& -\left(\begin{array}{c}
\hat{\Psi}(b) \\
\hat{\Psi}(a)
\end{array}\right)^{T} R^{T} W^{T} \tilde{W} R\left(\begin{array}{c}
\hat{\Psi}(b) \\
\hat{\Psi}(a)
\end{array}\right)
\end{aligned}
$$

which can be compactly written as

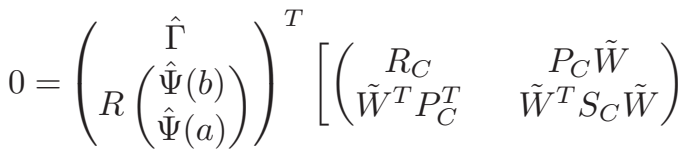

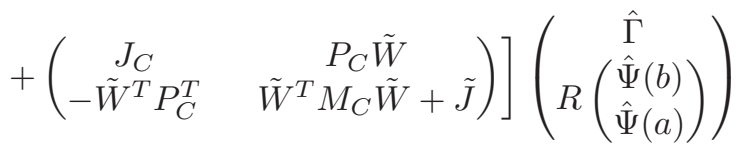

$$
\begin{aligned}
& -\frac{1}{2}\left(\begin{array}{c}
\hat{\Psi}(b) \\
\hat{\Psi}(a)
\end{array}\right)^{T}\left(\begin{array}{cc}
P_{1} & 0 \\
0 & -P_{1}
\end{array}\right)\left(\begin{array}{c}
\hat{\Psi}(b) \\
\hat{\Psi}(a)
\end{array}\right)
\end{aligned}
$$

once (2), (52), and (53) have been taken into account. Since for a skew-symmetric matrix $Q$ there holds that $v^{T} Q v=0$, we see that the middle term in above equality disappears. From (9) and (53), we see that the remaining two terms are non-negative. Hence, (54) implies that both terms are zero, thus (21) holds, and by (53) we conclude that (20) holds as well.

\section{ACKNOWLEDGMENT}

The hospitality and support of the National Engineering Institute in Mechanics and Microtechnologies (ENSMM) and the (AS2 M) FEMTO-ST Laboratory, Besançon, is gratefully acknowledged.

\section{REFERENCES}

[1] H. Paynter, Analysis and Design of Engineering Systems. Cambridge, MA: MIT Press, 1961.

[2] B. Maschke and A. van der Schaft, "Port controlled Hamiltonian systems: Modeling origins and system theoretic properties," in Proc. 3rd IFAC Symp. Control Systems (NOLCOS 1992), Bordeaux, France, Jun. 1992, pp. 282-288.

[3] A. van der Schaft, $L_{2}$-Gain and Passivity Techniques in Nonlinear Control, ser. Commun. and Control Eng. Springer-Verlag, New York, 2000.

[4] V. Duindam, A. Macchelli, S. Stramigioli, and H. Bruyninckx, Modeling and Control of Complex Physical Systems: The Port-Hamiltonian Approach. Springer, Berlin/Heidelberg, Germany, 2009.

[5] A. van der Schaft and D. Jeltsema, "Port-Hamiltonian systems theory: An introductory overview," Foundations and Trends in Systems and Control, vol. 1, no. 2-3, pp. 173-378, Jun. 2014.

[6] G. Golo, "Interconnection structures in port-based modeling: Tools for analysis and simulation," Ph.D. dissertation, University of Twente, Enschede, The Netherlands, 2002.

[7] A. van der Schaft and B. Maschke, "Hamiltonian formulation of distributed parameter systems with boundary energy flow," J. Geom. and Phys., vol. 42, no. 1-2, pp. 166-194, May 2002.

[8] Y. Le Gorrec, H. Zwart, and B. Maschke, "Dirac structures and boundary control systems associated with skew-symmetric differential operators," SIAM J. Control and Optimiz., vol. 44, no. 5, pp. 1864-1892, 2005.

[9] A. Macchelli and B. Maschke, Modeling and Control of Complex Physical Systems: The Port-Hamiltonian Approach. Springer, Berlin/ Heidelberg, Germany, 2009, ch. Infinite-Dimensional Port-Hamiltonian Systems, pp. 211-271.

[10] P. Olver, Application of Lie Groups to Differential Equations, 2nd ed. Springer-Verlag, Berlin/Heidelberg, Germany 1993.

[11] M. Schöberl and A. Siuka, "On Casimir functionals for infinitedimensional port-Hamiltonian control systems," IEEE Trans. Autom. Control, vol. 58, no. 7, pp. 1823-1828, Jul. 2013.

[12] H. Rodriguez, A. van der Schaft, and R. Ortega, "On stabilization of nonlinear distributed parameter port-controlled Hamiltonian systems via energy shaping," in Proc. 40th IEEE Conf. Decision and Control (CDC 2001), vol. 1, Dec. 2001, pp. 131-136.

[13] A. Macchelli and C. Melchiorri, "Modeling and control of the Timoshenko beam. The distributed port Hamiltonian approach," SIAM J. Control and Optimiz., vol. 43, no. 2, pp. 743-767, 2005. 
[14] A. Macchelli and C. Melchiorri, "Control by interconnection of mixed port Hamiltonian systems," IEEE Trans. Autom. Control, vol. 50, no. 11, pp. 1839-1844, Nov. 2005.

[15] R. Pasumarthy and A. van der Schaft, "Achievable Casimirs and its implications on control by interconnection of port-Hamiltonian systems," Int. J. Control, vol. 80, no. 9, pp. 1421-1438, 2007.

[16] A. Siuka, M. Schöberl, and K. Schlacher, "Port-Hamiltonian modelling and energy-based control of the Timoshenko beam," Acta Mechanica, vol. 222, no. 1-2, pp. 69-89, 2011.

[17] R. Ortega, A. van der Schaft, I. Mareels, and B. Maschke, "Putting energy back in control," IEEE Control Syst. Mag., vol. 21, no. 2, pp. 18-33, Apr. 2001.

[18] H. Zwart, Y. Le Gorrec, B. Maschke, and J. Villegas, "Well-posedness and regularity of hyperbolic boundary control systems on a one-dimensional spatial domain," ESAIM: Control, Optimiz., and Calculus of Variat., vol. 16, no. 4, pp. 1077-1093, Oct. 2010.

[19] R. Curtain and H. Zwart, An Introduction to Infinite Dimensional Linear Systems Theory. New York: Springer-Verlag, 1995.

[20] B. Jacob and H. Zwart, Linear Port-Hamiltonian Systems on Infinitedimensional Spaces, ser. Operator Theory: Advances and Applications. Basel, Switzerland: Birkhäuser, 2012, vol. 223.

[21] M. Dalsmo and A. van der Schaft, "On representation and integrability of mathematical structures in energy-conserving physical systems," SIAM J. Control and Optimiz., vol. 37, pp. 54-91, 1999.

[22] A. Macchelli, "Dirac structures on Hilbert spaces and boundary control of distributed port-Hamiltonian systems," Syst. \& Control Lett., vol. 68, pp. 43-50, Jun. 2014.

[23] A. Macchelli, "Passivity-based control of implicit port-Hamiltonian systems," SIAM J. Control and Optimiz., vol. 52, no. 4, pp. 2422-2448, 2014

[24] J. Villegas, H. Zwart, Y. Le Gorrec, B. Maschke, and A. van der Schaft, "Stability and stabilization of a class of boundary control systems," in Proc. 44th IEEE Conf. Decision and Control and European Control Conf. (CDC-ECC 2005), Dec. 12-15, 2005, pp. 3850-3855.

[25] J. Villegas, "A Port-Hamiltonian Approach to Distributed Parameter Systems," Ph.D. dissertation, University of Twente, Enschede, The Netherlands, 2007.

[26] A. Macchelli, "Boundary energy shaping of linear distributed portHamiltonian systems," Eur. J. Control, vol. 19, no. 6, pp. 521-528, Dec. 2013

[27] H. Ramírez, Y. Le Gorrec, A. Macchelli, and H. Zwart, "Exponential stabilization of boundary controlled port-Hamiltonian systems with dynamic feedback," IEEE Trans. Autom. Control, vol. 59, no. 10, pp. 2849-2855, Oct. 2014.

[28] R. Ortega, A. van der Schaft, B. Maschke, and G. Escobar, "Interconnection and damping assignment passivity-based control of port-controlled Hamiltonian systems," Automatica, vol. 38, no. 4, pp. 585-596, 2002.

[29] Z. Luo, B. Guo, and O. Morgul, Stability and Stabilization of Infinite Dimensional Systems with Applications. London, U.K.: Springer-Verlag, 1999.

[30] J. Villegas, H. Zwart, Y. Le Gorrec, and B. Maschke, "Exponential stability of a class of boundary control systems," IEEE Trans. Autom. Control, vol. 54, no. 1, pp. 142-147, Jan. 2009.

[31] W. Zhou, B. Hamroun, Y. Le Gorrec, and F. Couenne, "Infinite dimensional port Hamiltonian representation of chemical reactors," in Proc. 4th IFAC Workshop on Lagrangian and Hamiltonian Methods for Nonlinear Control (LHMNLC 2012), B. Maschke, C. Melchiorri, and A. van der Schaft, Eds. Bertinoro, Italy: University of Bologna, Aug. 29-31, 2012, pp. 1-6.

[32] A. van der Schaft, Modeling and Control of Complex Physical Systems: The Port-Hamiltonian Approach. Springer, Berlin/Heidelberg, Germany, 2009, ch. Port-Hamiltonian Systems, pp. 53-130.

[33] A. Macchelli, "Boundary energy shaping of linear distributed portHamiltonian systems," in Proc. 4th IFAC Workshop on Lagrangian and Hamiltonian Methods for Nonlinear Control (LHMNLC 2012), B. Maschke, C. Melchiorri, and A. van der Schaft, Eds. Bertinoro, Italy: University of Bologna, Aug. 29-31, 2012.

[34] Y. Le Gorrec, A. Macchelli, H. Ramírez, and H. Zwart, "Energy shaping of boundary controlled linear port Hamiltonian systems," in Proc. 19th IFAC World Congr., E. Boje and X. Xia, Eds. Cape Town, South Africa: Int. Feder. Autom. Control, Aug. 24-29 2014, pp. 1580-1585.

[35] A. Macchelli, "Boundary energy-shaping control of the shallow water equation," in Proc. 19th IFAC World Congr., E. Boje and X. Xia, Eds. Cape Town, South Africa: Int. Feder. Autom. Control, Aug. 24-29, 2014, pp. 1586-1591.

[36] G. Golo, V. Talasila, A. van der Schaft, and B. Maschke, "Hamiltonian discretization of boundary control systems," Automatica, vol. 40, no. 5, pp. 757-771, 2004.
[37] B. Maschke, C. Melchiorri, and A. van der Schaft, Eds., Proc. 4th IFAC Workshop on Lagrangian and Hamiltonian Methods for Nonlinear Control (LHMNLC 2012). Bertinoro, Italy, University of Bologna, Aug. 29-31, 2012.

[38] E. Boje and X. Xia, Eds., Proc. 19th IFAC World Congr.. Cape Town, South Africa: Int. Feder. Autom. Control, Aug. 24-29, 2014

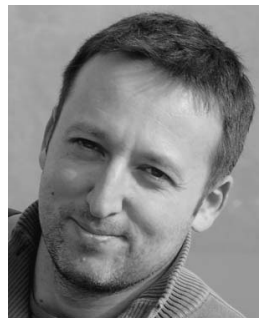

Alessandro Macchelli (M'06) received the Laurea degree (cum laude) and the Ph.D. degree in automatic control and operational research from the Department of Electronics, Computer Science and Systems (DEIS), University of Bologna, Bertinoro, Italy, in 2000 and 2003, respectively.

He was been a visiting scholar (sponsored by NACO2) at the University of Twente, Enschede, The Netherlands, in 2001 and a Post-Doc in 2003. In 2004, he was a Post-Doc with the Department of Electronics, Computer Science, and Systems (DEIS), University of Bologna, and in April 2005, he joined DEIS as Assistant Professor in robotics and industrial automation. After 2005, he was a Visiting Professor at Tongji University, Shanghai, China, at the Institute of Automatic Control and Control Systems Technology of the Johannes Kepler University, Linz, Austria), at the National Engineering Institute in Mechanics and MicroTechnologies (FEMTO-ST/AS2 M), Besaçon, France, and at the Institute of Cyber-Systems and Control, Zhejiang University, Hangzhou, China. His research activities are mainly focused on port-Hamiltonian systems, with particular emphasis on modelling, simulation and control of distributed parameter systems, and applications of the port-Hamiltonian framework to robotics and mechatronics. $\mathrm{He}$ is the author of more than 50 journal and conference papers on these topics.

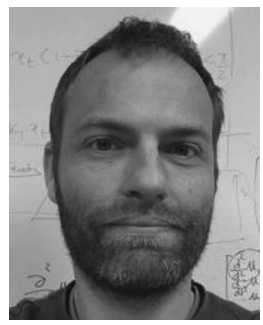

Yann Le Gorrec (M'14) received the Eng. degree in control, electronics, and computer engineering from the National Institute of Applied Sciences (INSA), Toulouse, France, in 1995, and the Ph.D. degree from the National Higher School of Aeronautics and Aerospace, Supaero, Toulouse, France, in 1998, with focus on robust control and self-scheduled controller synthesis.

From 1999 to 2008, he was an Associate Professor in automatic control at the Laboratory of Control and Chemical Engineering, Lyon Claude Bernard University (LAGEP), Villeurbanne, France. He has worked on port-Hamiltonian systems and their use for the modeling and control of irreversible and distributed parameter systems with an application to physico-chemical processes. Since September 2008, he has been a Professor at the National Engineering Institute in Mechanics and Microtechnologies. He is Deputy Director of the research department AS2 M of the FEMTO-ST Institute. His current field of research is the control of nonlinear and infinite-dimensional systems with an application to smart material based actuators and boundary controlled systems. $\mathrm{He}$ is the co-author of about 50 journal papers on these research topics.

Dr. Le Gorrec has been Co-Chair of the IFAC Technical Committee 2.6 on Distributed Parameter Systems since 2009, and since 2016, has been Chair of the IEEE Control Systems Society Technical Committee on Distributed Parameter Systems. 


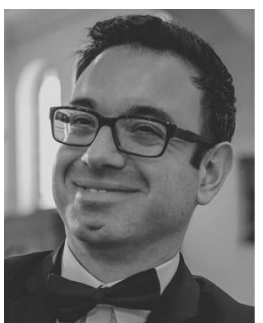

Héctor Ramírez (M'14) received the Eng. degree in electronic engineering and the M.Sc. degree in electrical engineering from the University of Concepción, Concepción, Chile, in 2006 and 2009, respectively. In 2012 he received the Ph.D. degree in automatic control from the University Claude Bernard and the Ph.D. in electrical engineering from the University of Concepción, for his work on nonlinear control of irreversible thermodynamic systems at the Laboratory of Control and Chemical Engineer-

ing (LAGEP), Lyon, France.

He held a post-doctoral position with the Department of Automatic Control and Micro Mechatronic systems (AS2 M) at the FEMTO-ST Research Institute, Besançon, France, from 2012 to 2013. Since 2013, he has been an Associate Professor in control engineering at the University of Bourgogne-Franche-Comté, Besançon, and affiliated with the AS2 M Department at FEMTO-ST. His research interests include portHamiltonian systems, modelling and control of multi-physical systems, and control of partial differential equations.

Dr. Ramírez is a member of the IFAC Technical Committee on nonlinear control systems (TC2.3) and the IEEE Control Systems Society Technical Committee on distributed parameter systems (DPS).

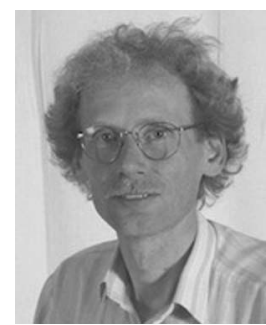

Hans Zwart (M'11) was born in HoogezandSappemeer, The Netherlands, in 1959. He received the Dr. degree in mathematics in 1984 and the Ph.D. degree in 1988, both from the University of Groningen, Groningen, The Netherlands. Both theses were written under the supervision of R. Curtain.

Since 1988, he has been with the Department of Applied Mathematics, University of Twente, Enschede, The Netherlands. He is the (co-) author of three books, one with R. Curtain which has become a standard reference on infinite-dimensional systems theory. $\mathrm{He}$ has published more than 70 research articles, treating various aspects of infinite-dimensional systems. His current research interests include stability, controllability, and control of infinite-dimensional systems, in particular for port-Hamiltonian systems. Since 2011, he also has a one-day per week position in the Dynamic and Control group, Department of Mechanical Engineering, Eindhoven University of Technology. 